\title{
ROTORCRAFT VIBRATION AND NOISE PREDICTION USING A UNIFIED AEROELASTIC ANALYSIS
}

\author{
D. PATT*, L. LiU*, \\ and P. P. FRIEDMANN ${ }^{\dagger}$ \\ Department of Aerospace Engineering \\ University of Michigan \\ Ann Arbor, Michigan 48109-2140 \\ email: peretzf@umich.edu
}

\begin{abstract}
A study of the combined helicopter noise and vibration problem was conducted. A refined aerodynamic model capable of calculating the unsteady surface pressure distribution on rotor blade/flap combinations is developed and coupled with an aeroelastic analysis simulation code. An acoustic prediction tool based on WOPWOP is combined with the aeroelastic analysis based on a flexible blade with coupled flap-lag-torsional dynamics. This unified analysis program is validated with experimental data. The acoustic environment of the helicopter is characterized by microphones mounted on the rotorcraft. Actively controlled flaps are used to reduce vibrations, and noise levels and acoustic signatures are monitored throughout this process. Changes in the properties of the individual bladevortex interactions are related to the acoustic results. This paper represents the careful theoretical treatment of the combined helicopter noise and vibration problem using actively controlled flaps for vibration reduction.
\end{abstract}

\section{$\underline{\text { Nomenclature }}$}

$\begin{array}{ll}\alpha_{R} & \text { Rotor shaft angle } \\ \alpha_{t p p} & \text { Tip-path plane angle } \\ \phi_{R} & \text { Lateral roll angle } \\ \gamma & \text { Lock number } \\ \mu & \text { Helicopter advance ratio } \\ \sigma & \text { Rotor solidity }\end{array}$

${ }^{*}$ Ph. D. Candidate, Student Member AIAA.

$\dagger$ François-Xavier Bagnoud Professor, Fellow AIAA.

Copyright (C) 2003 by D. Patt, L. Liu, and P. P. Friedmann. Published by the American Institute of Aeronautics and Astronautics, Inc. by permission.

\begin{tabular}{|c|c|}
\hline $\begin{array}{l}\theta_{0} \\
\theta_{1 s}, \theta_{1 c} \\
\theta_{t}\end{array}$ & $\begin{array}{l}\text { Collective pitch } \\
\text { Cyclic pitch components } \\
\text { Constant tail rotor pitch }\end{array}$ \\
\hline$\omega_{F 1} \omega_{T 1}^{\omega_{L 1}}$ & $\begin{array}{l}\text { Rotating fundamental blade frequen- } \\
\text { cies in flap, lead-lag and torsion, re- } \\
\text { spectively, nondimensionalized with } \\
\text { respect to } \Omega \text {. }\end{array}$ \\
\hline$\Omega$ & Rotor angular velocity \\
\hline$a_{0}$ & Lift curve slope \\
\hline$b$ & Blade semi-chord \\
\hline$c_{c}$ & Flap chord \\
\hline$C_{m 0}$ & Blade moment coefficient \\
\hline$C_{d 0}$ & $\begin{array}{l}\text { Blade drag coefficient in attached } \\
\text { flow }\end{array}$ \\
\hline $\begin{array}{l}\mathbf{C}_{\mathbf{0}}, \mathbf{C}_{1}, \mathbf{D} \\
\mathbf{E}, \mathbf{R}\end{array}$ & RFA coefficient matrices \\
\hline$C_{W}$ & Helicopter weight coefficient \\
\hline & Blade offset \\
\hline $\begin{array}{c}F_{H X 4}, F_{H Y} \\
F_{H Z 4}\end{array}$ & Nondimensional 4/rev hub shears \\
\hline $\mathbf{h}$ & Generalized motion vector \\
\hline$J_{R}$ & Sum of the trim residuals \\
\hline$L_{b}$ & Blade length \\
\hline$L_{c}$ & Control surface length \\
\hline $\begin{array}{c}M_{H X 4}, M_{H Y 4}, \\
M_{H Z 4}\end{array}$ & Nondimensional 4/rev hub moments \\
\hline$N_{b}$ & Number of blades \\
\hline$R$ & Rotor radius \\
\hline $\mathbf{T}$ & $\begin{array}{l}\text { Transfer matrix relating input har- } \\
\text { monics to vibrations }\end{array}$ \\
\hline $\mathbf{u}_{i}$ & Control input amplitudes \\
\hline$U(t)$ & $\begin{array}{l}\text { Air velocity relative to the blade sec- } \\
\text { tion }\end{array}$ \\
\hline $\mathbf{W}_{\mathbf{z}}$ & $\begin{array}{l}\text { Weighting matrix on vibration mag- } \\
\text { nitudes }\end{array}$ \\
\hline $\mathbf{W}_{\mathbf{u}}$ & Weighting matrix on control inputs \\
\hline $\mathrm{x}$ & $\begin{array}{l}\text { Aerodynamic attached flow state } \\
\text { vector }\end{array}$ \\
\hline
\end{tabular}




$\begin{array}{ll}X_{F A}, Z_{F A} & \begin{array}{l}\text { Longitudinal and vertical offsets be- } \\ \text { tween rotor hub and helicopter aero- } \\ \text { dynamic center }\end{array} \\ X_{F C}, Z_{F C} & \begin{array}{l}\text { Longitudinal and vertical offsets be- } \\ \text { tween rotor hub and helicopter cen- } \\ \text { ter of gravity } \\ \text { Vibration magnitudes }\end{array} \\ \mathbf{z}_{i} & \end{array}$

Introduction and Background

Vibration is one of the most critical concerns in the design of modern rotorcraft. Similarly, the generation of noise is another important consideration. Stricter demands for enhanced performance, comfort, and customer acceptance in new helicopters require simultaneous reduction of vibration and noise in helicopter designs.

In helicopters, the dominant source of vibrations is the rotor, which transfers vibrations to the rotor hub and fuselage at harmonics that are predominately $N_{b} /$ rev. Many methods, both passive and active, have been explored for the control of these loads. A description of a substantial amount of research on active vibration reduction can be found in a recent paper. ${ }^{1}$ Recently, actively controlled flaps (ACFs) have emerged as an efficient means of control of vibratory loads due to blade-vortex interaction (BVI) as well as alleviation of effect due to dynamic stall. ${ }^{2-7}$ Recent experimental results obtained from wind tunnel tests have demonstrated the potential of the ACF for reducing vibrations. ${ }^{8,9}$ Numerous other vibration reduction studies using ACFs have also been carried out. ${ }^{10,11}$ A survey of implementation of vibration reduction using ACFs employing adaptive materials-based actuation was presented in Ref. 12.

Among the active control approaches, two fundamentally different strategies have emerged: higher harmonic control (HHC) and individual blade control (IBC). Three different implementations of IBC have arisen: actuation at the blade root, the actively controlled flap, and active twist rotor blades. In each of these three cases, vibration is controlled by manipulating the unsteady aerodynamic loading in the rotating system. Recently, the HHC and IBC approaches that have been developed primarily for vibration reduction have also been studied as a means of reducing BVI noise. However, the control algorithms used are the same as those devised for vibration reduction.

Several wind tunnel tests of rotors have used the HHC approach for BVI noise control, and have showed reductions of about 5-6dB with open-loop control. ${ }^{13-17}$ The HART ${ }^{16}$ (higher-harmonic aeroacoustics rotor test) represents the most thorough study of HHC noise reduction to date. The HART wind-tunnel tests provide detailed acoustic results on a scaled BO-105 rotor using open-loop HHC, showing changes in noise or vibration levels due to harmonic input. Furthermore, noise data obtained from combined flight and wind-tunnel tests ${ }^{18}$ conducted on a MBB BO-105 complement HART data for validation purposes.

The IBC systems have also been tested as a noise reduction device in wind tunnels, on both the BO105 rotor $^{19-21}$ and the UH-60 rotor, ${ }^{22}$ and in flight tests on the BO-105. ${ }^{23,24}$ These tests suggest that noise reductions of $5-12 \mathrm{~dB}$ can be obtained by careful selection of open-loop control inputs. Recent flight tests of closed-loop IBC controllers have also demonstrated similar reductions. ${ }^{23,24}$ A thorough review of the early $\mathrm{HHC}$ and IBC noise reduction attempts is provided by $\mathrm{Yu}^{25,26}$ These tests have been augmented by several computational studies on noise reduction using the $\mathrm{HHC}$ approach ${ }^{27-30}$ and the IBC approach. ${ }^{24,31,32}$

Early tests with HHC revealed that inputs capable of reducing noise also increased vibration levels. ${ }^{13}$ Subsequent tests at both the Langley transonic dynamics tunnel (TDT) and the DNW (Duits Nederlandse wind tunnel) confirmed these observations. ${ }^{15,25,27}$ The HART Test ${ }^{16}$ showed that a $3 /$ rev input which produced a $6 \mathrm{~dB}$ noise reduction, also resulted in a $100 \%$ increase in vibratory loads. In applications of IBC, similar results were observed in the full-scale wind tunnel tests of a MBB BO-105 rotor conducted at NASA Ames in the $40 \times 80$ foot wind tunnel. ${ }^{19}$ When applied individually $3,4,5$ and $6 /$ rev components did not unilaterally decrease vibration and noise levels. In fact, $5 /$ rev inputs of 1 amplitude caused increases of more than $100 \%$ in vibration levels. Recent tests of a UH-60 helicopter in the NASA Ames $80 \times 120$ foot wind tunnel $^{22}$ also support this observation. It is noteworthy that the vibratory load increase that accompanies noise reduction appears to be a fundamental aspect of the problem since it appears in both configurations, even though the UH-60 rotor is much larger than the MBB BO-105 and has articulated blades. Flight tests conducted in Germany of the BO-105 also have produced somewhat similar results. ${ }^{31,33}$ Kube $^{33}$ notes that a single 2/rev input with a phase shift of $240^{\circ}$ yields a $6 \mathrm{~dB}$ BVI noise reduction but also increases $4 /$ rev vibrations by about $150 \%$. Very limited simultaneous vibration and noise reduction was obtained using multiple input harmonics ${ }^{19,20}$ on a BO-105 rotor. However the noise reduction 
(about $1 \mathrm{~dB}$ ) was not satisfactory, and investigation of the simultaneous reduction problem had a very limited scope.

Computational simulations have been unable to provide satisfactory insight on these experimental results, and attempts to explain the underlying physics have not been successful. In the HART test, ${ }^{16}$ it was mentioned that a decreased BVI missdistance contributed to lowered vibrations, but other studies suggest that changes in BVI inclination angle are more likely to be responsible for lowered vibrations. ${ }^{32}$ As implied from this review, further study is required to improve the fundamental understanding of the mechanism of simultaneous vibration and noise reduction.

The overall objective of this paper is to study the noise generated during the vibration reduction process implemented using single and dual ACF systems, throughout the flight envelope of a typical helicopter. The specific objectives of this study are: (1) development of a refined rotor aerodynamic model capable of generating unsteady time domain pressure distribution in the spanwise and chordwise directions along the blade that accounts for compressibility, free wake, and dynamic stall; (2) tight coupling between the aerodynamics and blade motion based on coupled flap-lag-torsional dynamics, including moderate deflections and trim; (3) development of a noise prediction capability based on WOPWOP ${ }^{34}$ which includes coupled flap-lagtorsional blade dynamics; (4) tracking of noise generation during the vibration reduction process using microphones located on and around the helicopter.

\section{Aeroelastic Model}

The present study is based upon an aeroelastic response analysis capable of modeling vibration reduction in rotorcraft using single and dual ACF systems. The code, which was gradually developed by Professor Friedmann and his students during the last decade, contains a fairly good unsteady aerodynamic model that consists of three primary components: (1) an unsteady, compressible, twodimensional aerodynamic model for the blade-flap combination that accounts for variations in the oncoming flow velocity, based upon rational function approximation (RFA) of aerodynamic loads; ${ }^{4}$ (2) a free wake model for the calculation of nonuniform inflow distributions; $;^{5,6}$ (3) a modified version of the ONERA dynamic stall model. ${ }^{7}$ This code has produced reasonably good correlation with wind tunnel tests conducted on a small-scale ACF system. ${ }^{5}$ The code was also used successfully to simulate alleviation of vibration effects due to dynamic stall as well as BVI on a rotor that resembles a BO-105 rotor.

\section{Structural Dynamic Model}

The structural dynamic model is directly taken from Ref. 3. The rotor is assumed to be composed of four identical hingeless blades, connected to a fixed hub, with an offset $e$, and rotates at constant RPM. The blade has fully coupled flap, lead-lag, and torsional dynamics, as shown in Fig.1. Small strains and moderate deflections are assumed. The inertial loads are obtained from D'Alembert's principle. The control surfaces are assumed to be an integral part of the blade, attached at a number of spanwise stations. It is assumed that the control surfaces do not modify the structural properties of the blade, only the inertial and aerodynamic loads due to the flap are accounted for.

\section{Aerodynamic Model}

\section{RFA Aerodynamics}

Blade section aerodynamic loads are calculated using RFA, an approach described by Myrtle and Friedmann. ${ }^{4}$ The RFA approach is an unsteady time-domain aerodynamic theory that accounts for compressibility, variations in the incoming flow and a combined blade and trailing edge flap configuration in the cross-section. The RFA approach produces an approximate transfer function between the generalized motion vector and the generalized attached flow force vector, as shown below:

$$
\begin{aligned}
\dot{\mathbf{x}}(t)= & \frac{U(t)}{b} \mathbf{R}(M) \mathbf{x}(t)+\mathbf{E}(M) \dot{\mathbf{h}}(t) \\
\mathbf{f}_{\mathbf{A}}(t)= & \frac{1}{U(t)}\left(\mathbf{C}_{0}(M) \mathbf{h}(t)+\mathbf{C}_{1}(M) \frac{b}{U(t)} \dot{\mathbf{h}}(t)\right. \\
& +\mathbf{D}(M) \mathbf{x}(t)) .
\end{aligned}
$$

\section{Free Wake Calculation}

A free wake is used to obtain the non-uniform inflow distribution. ${ }^{5,6}$ The free wake model has been extracted $^{35}$ from the comprehensive rotorcraft code CAMRAD/JA. ${ }^{36}$ The wake vorticity is created in the flow field as the blade rotates, and then convected with the local velocity of the fluid. The local velocity of the fluid consists of the free stream velocity, and the wake self-induced velocity. The wake 
geometry calculation contains the following ingredients: (1) the position of the blade generating the wake element is calculated, this is the point at which the wake vorticity is created; (2) the undistorted wake geometry is computed as wake elements are convected downstream from the rotor by the free stream velocity; (3) distortion of wake due to the wake self-induced velocity is computed and added to the undistorted geometry, to obtain a free wake geometry. The wake calculation ${ }^{36}$ is based on a vortex-lattice approximation for the wake. A free wake obtained from this approach is shown in Fig. 2 , for illustrative purposes.

\section{Dynamic Stall Model}

The third component of the aerodynamic model is a semi empirical dynamic stall model based on a modified version of the ONERA dynamic stall model $^{7}$ to produce a comprehensive simulation of the unsteady loading on the environment blade. The modified aerodynamic state vector for each blade section consists of RFA attached flow states and ONERA separated flow states, together with the representation of the free wake.

\section{Computation of Pressure Distribution}

For the noise prediction, the compressible unsteady pressure distribution on the helicopter blade in both chordwise and spanwise directions is required with sufficient resolution for acoustic computations is required. Therefore, the analysis and its implementation in the aeroelastic simulation code had to be modified to include this feature. The original implementation of the RFA approach ${ }^{4}$ was aimed only at producing the unsteady pressure, and aerodynamic load distribution in the spanwise direction. The unsteady chordwise pressure distribution was obtained by extending the RFA approach. The doublet-lattice method (DLM) was used to generate the chordwise pressure distribution in the frequency domain by introducing a number of chordwise panels. The pressure differentials at the middle of the panels were computed and tabulated at each chordwise station over a range of Mach numbers and reduced frequencies. Again, the RFA approach was used to convert the frequency domain aerodynamics into the time domain, using Eqs. 1 and 2. The influence coefficient matrices $\mathbf{C}_{\mathbf{0}}, \mathbf{C}_{\mathbf{1}}$ now correspond to the pressure distribution. Thus, the unsteady chordwise pressure distribution on the airfoil was obtained with the required resolution. In this approach, aerodynamic states for the pressure differen- tial at each chordwise station were introduced in the calculation of the pressure distribution. However, these states were not required in the coupled helicopter trim and aeroelastic response solution procedure, because the sectional loads determining blade response are calculated separately. The block diagram in Fig. 3 describes the pressure distribution computation and its coupling with the aeroelastic analysis procedure.

The oscillatory chordwise pressure distribution generated at the blade station when using the DLM approach (the first block of Fig. 3) yields an unrealistically high pressure distribution in the leading edge region. This is the well-known leading edge singularity problem, which has been studied in Ref. 37 using both experimental data and computational fluid dynamics (CFD). The effect of this pressure singularity on the aerodynamic force resultants (lift and moment) is small, however its effect on noise could be significant. To remedy this situation without using costly CFD computations, a physically justifiable limit has been introduced into the code. This limit is known as the vacuum pressure limit (VPL). ${ }^{38,39}$ The VPL utilizes physical insight to argue that the resultant pressure differential between the atmosphere and moving airfoil must always be positive, which implies that the magnitude of the leading edge suction cannot exceed atmospheric pressure. The pragmatic advantage of this approach is that it produces reasonable results with minimal computational cost.

\section{BVI Detection and Visualization}

Blade-vortex interaction, its detection and visualization are important for understanding the mechanism of noise generation during the active control of vibrations. A methodology for detecting helicopter blade vortex interactions was implemented, using the planar detection method. This method was described in detail in Ref. 40. A plane is defined along the rotor blade, perpendicular to the plane of the rotor. When any tip vortex segment as computed from the free wake routine intersects this plane and is within a specified height above or below the blade, a BVI event is recorded. The miss distance between the vortex segment and the blade and the interaction angle between the vortex segment and the blade are then computed and stored. With this data, properties of each intersection event (to the limit of the wake routine resolution) can be analyzed. Figure 4 depicts this procedure. 


\section{$\underline{\text { Acoustic Formulation }}$}

The helicopter noise prediction code WOP$\mathrm{WOP}^{41}$ is based on Farassat's formulation $1 \mathrm{~A},{ }^{42}$ which is derived from the Ffowcs-Williams Hawkings equation with the neglect of the quadrupole term. ${ }^{43}$ This formulation is valid for arbitrary blade motion and geometry. The original version of WOPWOP requires blade harmonics and surface loading as input, which can be provided by either experiments or a suitable helicopter analysis code. The version of WOPWOP that requires blade harmonics and surface loading input from a rotor analysis code contains the assumption of an offset hinged rigid blade and proceeds to calculate the acoustic information based on this assumption. This assumption is incompatible with the elastic blade model described in the previous section. To remedy this situation, the blade dynamics in WOPWOP were replaced by a fully flexible blade model with partial span trailing edge flaps. This was accomplished by discretizing the blade into a number of individual panels as shown in Fig.5. The time domain response of each of these panels was obtained from the aeroelastic response analysis. This information, together with the unsteady pressure distribution on the panel, calculated as described earlier, serves as the basis of the acoustic computations.

\section{Aeroelastic Response Solution}

The blade is discretized ${ }^{3}$ using the global Galerkin method, based upon the free vibration modes of the rotating blade. Three flapping modes, two lead-lag modes and two torsional modes are used in the actual implementation. The combined structural and aerodynamic equations form a system of coupled differential equations that can be cast in state variable form. They are then integrated in the time domain using the Adams-Bashforth DE/STEP predictorcorrector algorithm.

\section{Trim Procedure}

The propulsive trim procedure ${ }^{35}$ enforces three force equations (longitudinal, vertical, and lateral forces) and three moment equilibrium equations (roll, pitch, and yaw moments). A simplified tail rotor model is used, using uniform inflow and blade element theory. The six trim variables are the rotor shaft angle $\alpha_{R}$, the collective pitch $\theta_{0}$, the cyclic pitch $\theta_{1 s}$ and $\theta_{1 c}$, the tail rotor constant pitch $\theta_{t}$ and lateral roll angle $\phi_{R}$. The trim procedure is based on the minimization of the sum $J_{R}$ of the trim residuals. At higher advance ratios $(\mu>0.30)$, a more refined version of the trim procedure which facilitates convergence is used. ${ }^{7} \mathrm{~A}$ wind tunnel trim procedure was also implemented for validation with HART, wherein zero pitch and roll moment conditions are enforced.

\section{Control of Vibrations}

The approach to active control of vibrations using the ACF system has been described in earlier papers. ${ }^{2,3,6,7}$ The control algorithm used in these studies and others aimed at vibration reduction employs a control algorithm developed more than two decades ago for HHC application to vibration reduction. The algorithm is based on a linear, quasistatic, frequency domain representation of vibratory response to control inputs. The input harmonics of the ACF, which consist of $2,3,4$ and $5 /$ rev contributions are related to the vibration magnitudes through a transfer matrix $\mathbf{T}$, given by

$$
\mathbf{T}=\frac{\partial \mathbf{z}_{\mathbf{i}}}{\partial \mathbf{u}_{\mathbf{i}}}
$$

The control strategy is based on the minimization of a performance index described in $\mathrm{in}^{2-7,44}$ that is a quadratic function of the vibration magnitudes $\mathbf{z}_{i}$ and control input amplitudes $\mathbf{u}_{i}$ :

$$
J=\mathbf{z}_{i}^{T} \mathbf{W}_{\mathbf{z}} \mathbf{z}_{i}+\mathbf{u}_{i}^{T} \mathbf{W}_{\mathbf{u}} \mathbf{u}_{i},
$$

The subscript $i$ refers to the $i$ th control step, reflecting the discrete-time nature of the control. The time interval between each control step must be long enough to allow the system to return to the steady state so that the $4 /$ rev vibratory magnitudes can be accurately measured. The matrices $\mathbf{W}_{\mathbf{z}}$ and $\mathbf{W}_{\mathbf{u}}$ are weighted matrices on the vibration magnitude and control input, respectively.

The optimal control is:

$$
\mathbf{u}_{i}^{*}=-\mathbf{D}^{-1} \mathbf{T}^{T}\left\{\mathbf{W}_{\mathbf{z}} \mathbf{z}_{i-1}-\mathbf{W}_{\mathbf{z}} \mathbf{T} \mathbf{u}_{i-1}\right\}
$$

where

$$
\mathbf{D}=\mathbf{T}^{T} \mathbf{W}_{\mathbf{z}} \mathbf{T}+\mathbf{W}_{\mathbf{u}}
$$

It is important to emphasize that this classical algorithm has been recently modified so as to avoid excessive flap angles, and actuator saturation, ${ }^{45}$ and the version of the algorithm in the code contains this modification.

It should be noted that the HART study, like others attempting noise reduction in open-loop or 
closed-loop modes, have tried to use this same algorithm, although developed for vibration reduction. It is not surprising that this approach has been only partially successful in reducing noise, since no measure of noise reduction has been added to the objective function.

\section{Validation}

\section{Wake Routine and BVI Detection}

Beaumier and Spiegel ${ }^{29}$ conducted a validation of their MESIR (Mise en Equilibre du Sillage Rotor) wake geometry routine against results from the HART Test. ${ }^{16}$ Helicopter and rotor data for the HART Test are presented in Table 1. The MESIR code provided prediction of blade-vortex interaction events, shown in Figure 6. The HART test case selected corresponds to the following conditions: $\alpha_{R}=5.3^{\circ}$ and $\alpha_{t p p}=3.8^{\circ}$, simulating a $6^{\circ}$ descent.

To validate the current simulation, the same flight condition was reproduced. The predicted bladevortex interactions are shown in Figure 6. Unfortunately, the current study was limited to a wake resolution of $15^{\circ}$, a restriction that will removed in subsequent studies. Also, the HART Test rotor used a NACA 23012 airfoil, while the airfoil in the current study is a NACA 0012. Despite these limitations, good agreement with ONERA results was obtained.

\section{Blade Tip Deflections}

Tauszig $^{46}$ has performed validation of an aeroelastic simulation code with HART for a flight condition whose principal parameters have been provided in Table 1. The blade tip deflections predicted by our code were compared in Fig. 7 with the results obtained in Ref. 46 together with the experimentally measured deflections from the HART test. Although no simulation has been able to match all of the features of the HART profile exactly, ${ }^{46}$ Fig. 7 shows that the current simulation reproduces many of the HART features and, at the same time, it also yields tip deflection values that are quite close to the HART results.

\section{$\underline{\text { Results }}$}

In this study, a helicopter configuration resembling a MBB BO-105 helicopter with a four-bladed hingeless rotor system was used. The results were obtained using a propulsive trim procedure. The data used in the computations is summarized in Table 2 . The characteristics of the actively controlled flap configuration are shown in Table 3. The acoustic environment in the vicinity of the helicopter was obtained by assuming that microphones capable of measuring the required noise information are located at the points depicted in Fig. 8. Microphones were placed on a boom extending from the nose (labeled NOSE), on the landing skid (labeled SKID), at the carpet plane on the retreating side (labeled RETR), and on the advancing side (labeled ADV1 and ADV2 ).

\section{Influence of Vibration Reduction on Noise}

The effect of vibration reduction on noise generation is examined by considering first a flight condition of $\mu=0.15$ with a descent angle of $6^{\circ}$. Carpet plots showing the noise production below the rotorcraft are given in Fig. 9. The baseline case shows three major noise peaks characterizing noise directivity: one in front of the rotor, one on the advancing side, and one on the retreating side. After vibration reduction, the carpet plot shows that all noise levels go up by five or more $\mathrm{dB}$ except in some areas on the retreating side. Moreover, the carpet plot also shows that the advancing side is most affected, with the entire first quadrant experiencing an increase in noise of about $10 \mathrm{~dB}$. The retreating side noise levels remain almost the same or decrease slightly.

Noise levels at various locations during the vibration reduction process are shown in Fig. 10. The skid microphone records an $11 \mathrm{~dB}$ increase. The advancing side microphones register increases of $4 \mathrm{~dB}$ and $7 \mathrm{~dB}$, respectively, while the retreating side microphone shows a $4 \mathrm{~dB}$ increase. Although the microphone on the nose boom shows a $9 \mathrm{~dB}$ reduction in noise, on the lower carpet plane the same $x-y$ point shows a $6 \mathrm{~dB}$ increase, suggesting that the near acoustic field differs substantially from the plane below.

The results for the 4/rev vibratory hub shears and moments during the active control of the vibrations are shown in Fig. 11. The controller, whose performance is governed by an objective function aimed at vibration reduction, effectively reduces vibratory loads during descent. Vibratory hub shears fall between $30-60 \%$ compared to the baseline values. The most important vibratory component, the vertical hub shear FHZ4, is reduced by nearly $60 \%$.

To enhance our understanding of BVI during vibration reduction, the changes in blade-vortex inter- 
action as represented by the miss distance and interaction angle in the rotor plane were computed and are displayed in Fig. 12 and 13, respectively. The miss distance characteristics change noticeably after vibration reduction, with closer interactions occuring near the hub on the advancing side, as shown in in Fig. 12. The BVI interaction angles also change after vibration control, as shown in Fig. 13. On the advancing side, in the first quadrant, the interactions become closer to parallel. This is thought to be responsible for the increased advancing side noise.

The same rotor was also considered at a higher advance ratio, $\mu=0.30$, without changing the angle of descent, and the results are shown in Fig. 14. Vibration levels are similar to those at $\mu=0.15$, with higher blade loading offsetting decreased wake interaction. Again, the vertical hub shear FHZ4, is reduced by about $60 \%$. The changes in noise levels are shown in Fig. 15. The skid microphone shows a $7 \mathrm{~dB}$ increase in noise level after vibration reduction, and the nose noise level increases by $4 \mathrm{~dB}$. The advancing side microphones show 3 and $5 \mathrm{~dB}$ increases, respectively. However, the retreating side noise is reduced by almost $4 \mathrm{~dB}$ after vibration reduction. These results imply that the directivity of the noise is affected in a manner that resembles the $\mu=0.15$ case, with advancing side blade noise increasing most.

\section{Effect of Blade Flexibility}

Several previous computational acoustic studies on rotorcraft have input blade pressure to the acoustic code using only an offset-hinged spring-restrained blade model for acoustic computation. ${ }^{47,48}$ Thus, for noise prediction purposes, the blade is modeled as a rigid rod. It is therefore important to determine the effect of such an assumption on the acoustic output. A configuration resembling a MBB BO-105 rotor was considered at $\mu=0.15$ and a $6^{\circ}$ rate of descent. The acoustic signature at the SKID point was monitored and is shown schematically in Fig. 16. Figure 16 depicts a comparison of the acoustic pressure distribution on the offset-hinged spring-restrained blade, with the same quantity on the flexible blade represented by seven rotating modes. The character and magnitude of the noise differs substantially, depending on the blade dynamic model used. Clearly, the accurate representation of blade dynamics, coupling, and geometrical nonlinearities has a substantial effect on the local unsteady pressure distribution and associated noise. Therefore, blade dynamics and flexibil- ity must be properly incorporated in any acoustic simulation.

\section{Conclusions}

A numerical simulation of noise generation during closed-loop vibration reduction using actively controlled flaps has been conducted. A refined aerodynamic model capable of producing unsteady pressure distribution along the blade was coupled with a modified version of the WOPWOP acoustic prediction tool. A single ACF configuration was used to reduce $4 / \mathrm{rev}$ vibrations, and the accompanying noise modifications were carefully examined. Only a limited number of numerical results have been obtained in the course of this study. The principal conclusions that can be gleaned from these results are summarized below:

1. The ACF configuration is a useful vibration reduction device for descending flight in the presence of wake.

2. Significant noise increases accompany vibration reduction, especially in the first quadrant. The characteristics of the acoustic environment change during the reduction of vibrations by active control. This suggests that the nature of BVI interactions change due to vibration reduction.

3. Blade flexibility and coupled blade dynamics are important parameters that have to be properly incorporated in a noise prediction code.

4. It is important to note that one should not be surprised by noise increases during active vibration reduction, since the control algorithms used contain only vibration levels in the objective functions.

\section{Acknowledgments}

This research was supported in part by the NASA Graduate Student Researcher Program NASA NGT 2-52269 for D. Patt, with Dr. S. Jacklin, NASA Ames Research Center, as grant monitor and by ARO grant 02-1-0202 with Dr. G. Anderson as grant monitor. Partial support by the FXB Center for Rotary and Fixed Wing Air Vehicle Design is also gratefully acknowledged.

\section{REFERENCES}

[1] Friedmann, P. P. , "Rotary Wing Aeroelasticty - Current Status and Future Trends," Proceedings of the 39th AIAA Aerospace Sciences Met- 
ting and Exhibit, Reno, NV, May 2001. AIAA Paper No. 2001-0427.

[2] Millott, T. A. and Friedmann, P. P. , "Vibration Reduction in Rotorcraft Using Active Control: A Comparison of Various Approaches," Journal of Guidance, Control, and Dynamics, Vol. 18, 1995, pp. 664-673.

[3] Millott, T. A. and Friedmann, P. P. , Vibration Reduction in Helicopter Rotors Using an Actively Controlled Partial Span Trailing Edge Flap Located on the Blade. NASA CR 4611, Jun. 1994.

[4] Myrtle, T. and Friedmann, P. P. , "Application of a New Compressible Time Domain Aerodynamic Model to Vibration Reduction in Helicopters Using an Actively Controlled Flap," Journal of the American Helicopter Society, Vol. 46, No. 1, Jan. 2001, pp. 32-43.

[5] de Terlizzi, M. and Friedmann, P. P. , "Active Control of BVI Induced Vibrations Using a Refined Aerodynamic Model and Experimental Correlation," Proceedings of the 51st Annual Forum of the American Helicopter Society, Fort Worth, TX, May 1995, pp. 510-524.

[6] Friedmann, P. P. , de Terlizzi, M. , and Myrtle, T. , "New Developments in Vibration Reduction with Actively Controlled Trailing Edge Flaps," Mathematical and Computer Modelling, Vol. 33, 2001, pp. 1055-1083.

[7] Depailler, G. and Friedmann, P. P. , "Reductions of Vibrations Due to Dynamic Stall in Helicopters Using an Actively Controlled Flap," Proceedings of the 43rd AIAA/ASME/ASCE/AHS/ACS Structures, Structural Dynamics and Materials Conference, Denver, CO, Apr. 2002.

[8] Fulton, M. and Ormiston, R. A. , "Small-Scale Rotor Experiments with On-Blade Elevons to Reduce Blade Vibratory Loads in Forward Flight," Proceedings of the 54th Annual Forum of the American Helicopter Society, Washington, DC, May 1998, pp. 433-451.

[9] Koratkar, N. A. and Chopra, I. , "Wind Tunnel Testing of a Smart Rotor Model with Trailing Edge Flaps," Journal of the American Helicopter Society, Vol. 47, No. 4, Oct. 2002, pp. $263-272$.
[10] Straub, F. K. and Charles, B. D. , "Aeroelastic Analysis of Rotors with Trailing Edge Flaps Using Comprehensive Codes," Journal of the American Helicopter Society, Vol. 46, No. 3, Jul. 2001, pp. 192-1991.

[11] Chopra, I. , Milgram, J. , and Straub, F. K. , "Comprehensive Rotorcraft Aeroelastic Analysis with Trailing Edge Flaps," Proceedings of the 52nd Annual Forum of the American Helicopter Society, Washington, D.C., Jun. 1996, pp. $715-725$.

[12] Chopra, I. , "Status of Application of Smart Structures Technology to Rotorcraft Systems," Journal of the American Helicopter Society, Vol. 45, No. 4, Oct. 2000, pp. 228-252.

[13] Brooks, T. F. , Booth, E. R. , Jolly, J. R., Yeager, W. , and Wilbur, M. , "Reduction of BladeVortex Interaction Noise Through Higher Harmonic Pitch Control," Journal of the American Helicopter Society, Vol. 35, No. 1, Jan. 1990, pp. 86-91.

[14] Brooks, T. F. and Booth, E. R. , "The Effect of Higher Harmonic Pitch Control on BladeVortex Interaction Noise and Vibration," Journal of the American Helicopter Society, Vol. 38, No. 3, Jul. 1993, pp. 45-55.

[15] Spletstoesser, W. , Schultz, K. J. , Kube, R. , Brooks, T. F. , Booth, E. R. , Niesl, G. , and Streby, O., "A Higher Harmonic Control Test in the DNW to Reduce Impulsive BVI Noise," Journal of the American Helicopter Society, Vol. 39, No. 4, Oct. 1994, pp. 3-13.

[16] Spletstoesser, W. , Kube, R. , Wagner, W. , Seelhorst, U. , Boutier, A. , Micheli, F. , Mercker, E. , and Pengel, K. , "Key Results From a Higher Harmonic Control Aeroacoustic Rotor Test (HART)," Journal of the American Helicopter Society, Vol. 42, No. 1, Jan. 1997, pp. 58-78.

[17] Yu, Y. H. , Tung, C. , van der Wall, B. , Pausder, H. J. , Burley, C. , Brooks, T. , Beaumier, P. , Delrieux, Y. , Mercker, E. , and Pengel, K. , "The HART-II Test: Rotor Wakes and Aeroacoustics with HigherHarmonic Pitch Control (HHC) Inputs - The Joint German/French/Dutch/US Project -," Proceedings of the 58th Annual Forum of the American Helicopter Society, Montreal, Canada, Jun. 2002. 
[18] Signor, D. B. and Watts, M. E. , "Blade-Vortex Interaction Noise: A Comparison of In-Flight, Full-Scale, and Small-Scale Measurements," Proceedings of the American Helicopter Society Aeromechanics Specialists Conference, San Francisco, CA, Jan. 1994, pp. 1.3.1-1.3.39.

[19] Jacklin, S. A. , "Second Test of a Helicopter Individual Blade Control System in the NASA Ames 40 by 80 Foot Wind Tunnel," Proceedings of the 2nd International American Helicopter Society Aeromechanics Specialists Conference, Bridgeport, CT Oct. 11-13, 1995.

[20] Swanson, S. M. , Jacklin, S. A. , Blaas, A. , Niesl, G. , and Kube, R. , "Reduction of Helicopter BVI Noise, Vibration, and Power Consumption through Individual Blade Control," Proceedings of the 51st Annual Forum of the American Helicopter Society, Fort Worth, TX, May 1995, pp. 662-680.

[21] Jacklin, S. A., Blaas, A. , Teves, D. , and Kube, R. , "Acoustic Results from a Full-Scale Wind Tunnel Test Evaluating Individual Blade Control," Proceedings of the 51st Annual Forum of the American Helicopter Society, Fort Worth, TX, May 1995, pp. 461-480.

[22] Jacklin, S. A. , Haber, A. , de Simone, G. , Norman, T. , Kitaplioglu, C. , and Shinoda, P. , "Full-Scale Wind Tunnel Test of an Individual Blade Control System for a UH-60 Helicopter," Proceedings of the 51st Annual Forum of the American Helicopter Society, Montreal, Canada, Jun. 2002.

[23] Bebesel, M. , Roth, D. , Dietrich, Pongratz, and Kube, R. , "Individual Blade Root Control Demonstration Recent Activities," Proceedings of the 27th European Rotorcraft Forum, Moscow, Russia, Sep. 2001, pp. 33.133.14 .

[24] Bebesel, Roth, and Pongratz, "Reduction of BVI Noise Ground - In Flight Evaluation of Closed-Loop Controller," Proceedings of the 28th European Rotorcraft Forum, Bristol, England, Sep. 2002, pp. 19.1-19.9.

[25] Yu, Y. H. , Brooks, T. F. , and Splettstoesser, W. , "Reduction of Helicopter Blade-Vortex Interaction Noise by Active Rotor Control Technology," Progress in Aerospace Sciences, Vol. 33, No. 10, Sep. 1997, pp. 647-687.
[26] Yu, Y. H. , "Rotor Blade-Vortex Interaction Noise," Progress in Aerospace Sciences, Vol. 36, No. 2, Feb. 2000, pp. 97-115.

[27] Brooks, T. , Booth, T. , Splettstoesser, W. , and Kube, R. , "Analysis of a Higher Harmonic Control Test to Reduce Blade Vortex Interaction Noise," AIAA Journal, Vol. 31, 1994, pp. $1341-1348$.

[28] van der Wall, B. , "Simulation of $\mathrm{HHC}$ on Helicopter Rotor BVI Noise Emission Using a Prescribed Wake Method," Proceedings of the 26th European Rotorcraft Forum, The Hague, Netherlands, Sep. 2000, pp. 43.2-43.15.

[29] Beaumier, P. and Spiegl, P. , "Validation of ONERA Aeroacoustic Prediction Methods for Blade-Vortex Interaction Using HART Test Results," Proceedings of the 51st Annual Forum of the American Helicopter Society, Fort Worth, TX, May 1995, pp. 510-524.

[30] Kube, R. and Splettstoesser, W. , "HHC Aeroacoustic Rotor Tests in the German-Dutch Wind Tunnel: Improving Physical Understanding and prediction results," Aerospace Science and Technology, Vol. 2, No. 3, Mar. 1998, pp. 177-190.

[31] Splettstoesser, W. , van der Wall, B. , and Schultz, F. , "The Effect of Individual Blade Pitch Control of Flight Test and Simulation Results," Proceedings of the 24th European Rotorcraft Forum, Marseilles, France, Sep. 1998, pp. AC07.1-AC07.15.

[32] Malovrh, B. and Gandhi, F. , "Localized Individual Pitch Control for Reduction of Helicopter Blade-Vortex Interaction Noise," Proceedings of the 28th European Rotorcraft Forum, Bristol, England, Sep. 2002, pp. 6.1-6.10.

[33] Kube, R. , van der Wall, B. , Schultz, and Splettstoesser, W. , "IBC Effects on BVI Noise and Vibrations a Combined Numerical and Experimental Investigation," Proceedings of the 55th Annual Forum of the American Helicopter Society, Montreal, Canada, May 1999.

[34] Brentner, K. , Prediction of Helicopter Rotor Discrete Frequency Noise: A Computer Program Incorporating Realistic Blade Motions and an Advanced Acoustic Formulation. NASA Technical Memorandum, Vol. 87721, 1986. 
[35] de Terlizzi, M. , "Blade Vortex Interaction and Its Alleviation Using Passive and Active Control Approaches," Ph.D. Dissertation, UCLA, 1999.

[36] Johnson, W. , A Comprehensive Analytical Model of Rotorcraft Aerodynamics and Dynamics, Vol. I: Theory Manual, Palo Alto, CA, Johnson Aeronautics, 1988.

[37] Roughen, K. , Baker, M. , and Fogarty, T. "Computational Fluid Dynamics and DoubletLattice Calculation of Unsteady Control Surface Aerodynamics," Journal of Guidance, Control, and Dynamics, Vol. 24, No. 1, Jan. 2001, pp. 160-166.

[38] Carlson, H. , Estimation of Attainable LeadingEdge Thrust for Wings at Subsonic and Supersonic Speeds. NASA TP-1500, Aug. 1979.

[39] Carlson, H. , Improved Method for Prediction of Attainable Wing Leading-Edge Thrust. NASA TP-3557, Apr. 1996.

[40] Gandhi,F. and Tauszig,L., "A Critical Evaluation of Various Approaches for the Numerical Detection of Helicopter Blade-Vortex Interactions," Journal of the American Helicopter Society, Vol. 45, No. 3, Jul. 2000, pp. 179-190.

[41] Brentner, K. S. , "Prediction of Helicopter Rotor Discrete Frequency Noise - A Computer Program Incorporating Realistic Blade Motions and Advanced Acoustic Formulation," NASA Technical Memorandum, Vol. 87721, 1986.

[42] Farassat, F. and Succi, G. P. , "The Prediction of Helicopter Rotor Discrete Frequency Noise," Vertica, Vol. 7, No. 4, Oct. 1983, pp. 309-320.

[43] Ffowcs Williams, J. E. and Hawkings, D. L. , "Sound Generation by Turbulence and Surfaces in Arbitrary Motion," Philosophical Transactions of Royal Soc. London, Series A, Vol. 264, No. 1151, May 1969, pp. 321-342.

[44] Johnson, W. , Self-Tuning Regulators for Multicyclic Control of Helicopter Vibrations. NASA Technical Paper 1996, 1982.

[45] Cribbs, R. and Friedmann, P. P. , "Actuator Saturation and Its influence on Vibration Reduction by Actively Controlled Flaps," Proceedings of the 42nd AIAA/ASME/ASCE/AHS/ACS Structures,
Structural Dynamics and Materials Conference, Seattle, WA, Apr. 2001. AIAA Paper No. 2001-1467.

[46] Tauszig, L. , "Analysis of Helicopter BladeVortex Interaction Noise With Application to Adaptive-Passive and Active Alleviation Methods," Ph.D. Dissertation, The Pennsylvania State University, 2002.

[47] Boyd, D. D. , Brooks, T. F. , Burley, C. L. , and Jolly, J. R. , "Aeroacoustic Codes for Rotor Harmonic and BVI Noise - Camrad.Mod1/HIRES," Journal of the American Helicopter Society, Vol. 45, No. 2, Apr. 2000, pp. 63-89.

[48] Wachspress, D. and Quackenbush, T. , "BVI Noise Prediction Using a Comprehensive Rotorcraft Analysis," Proceedings of the American Helicopter Society 57th Annual Forum, Washington, DC, May 2001.

Table 1: HART test configuration

\begin{tabular}{ll}
\hline$\underline{\text { Rotor Data }}$ & \\
\hline$N_{b}=4$ & $2 b=0.0605 R$ \\
$\omega_{F 1}=1.122$ & $\omega_{T 1}=3.93$ \\
$\omega_{F 2}=3.153$ & $\omega_{T 2}=11.45$ \\
$\sigma=0.077$ & \\
\hline $\begin{array}{l}\text { Helicopter Data } \\
C_{W}=0.0044\end{array}$ & $\mu=0.15$ \\
\hline
\end{tabular}

Table 2: Elastic blade configuration

\begin{tabular}{ll}
\hline$\underline{\text { Rotor Data }}$ & \\
$N_{b}=4$ & $2 b=0.05498 L_{b}$ \\
$\omega_{F 1}=1.123$ & $C_{d o}=0.01$ \\
$\omega_{L 1}=0.732$ & $C_{m o}=0.0$ \\
$\omega_{T 1}=3.17$ & $a_{o}=2 \pi$ \\
$\gamma=5.5$ & $\sigma=0.07$ \\
Helicopter Data & \\
$\overline{C_{W}=0.00515}$ & \\
$X_{F A}=0.0$ & $Z_{F A}=0.3$ \\
$X_{F C}=0.0$ & $Z_{F C}=0.3$ \\
\hline
\end{tabular}


Table 3: Flap configuration

\begin{tabular}{ll}
\hline$c_{c}=0.50 b$ & \\
Single Flap & \\
$x_{c}=0.75 L_{b} \quad L_{c}=0.12 L_{b}$
\end{tabular}

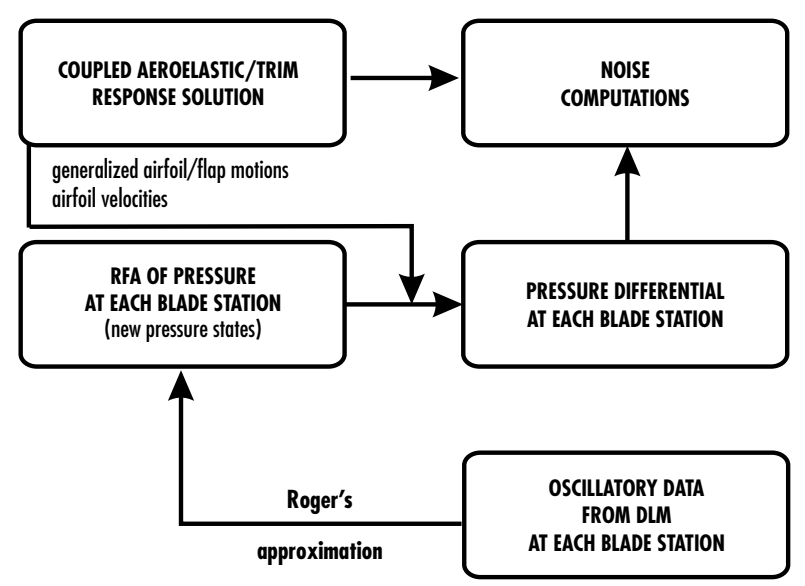

Figure 3: Block Diagram for pressure distribution computation

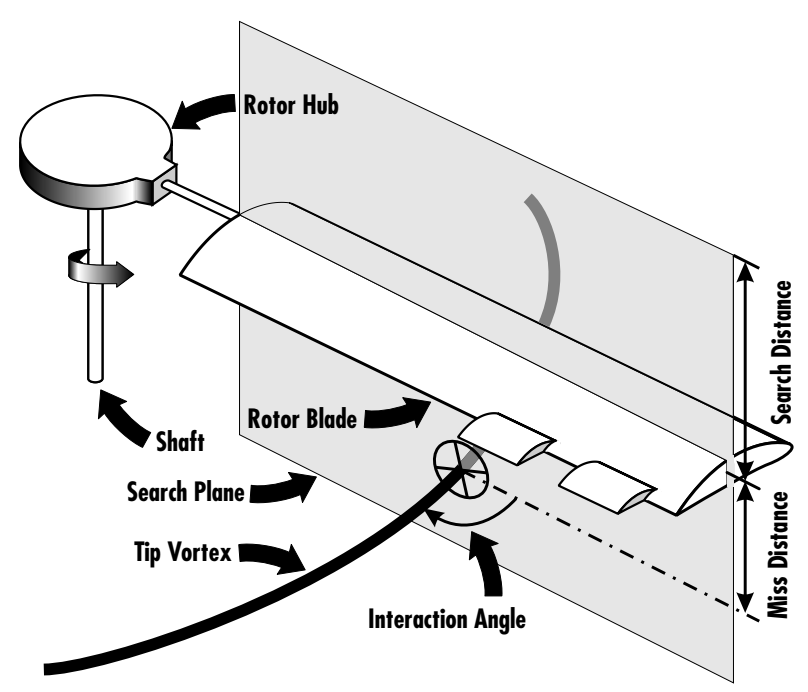

Figure 4: Planar detection of BVI intersections

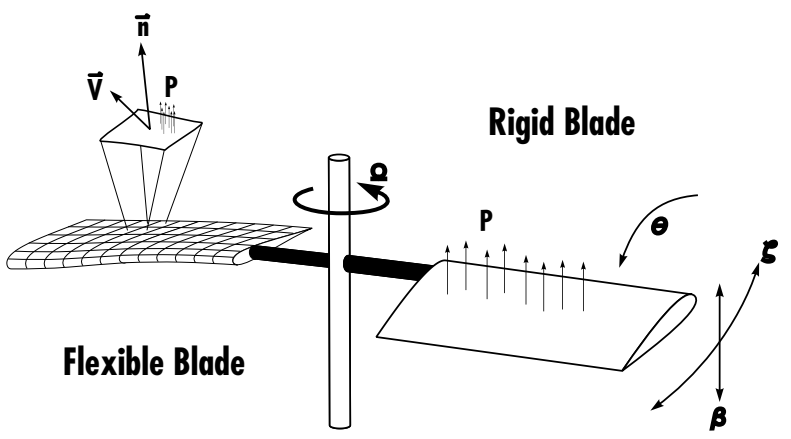

Figure 5: Rigid and flexible blade representations 


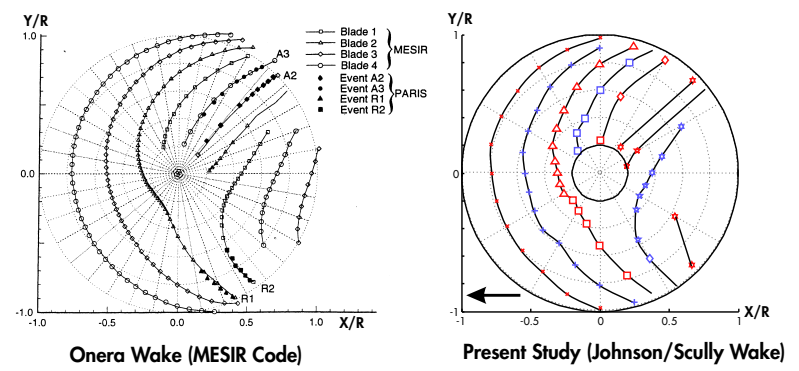

Figure 6: Validation of the wake model and BVI detection routine
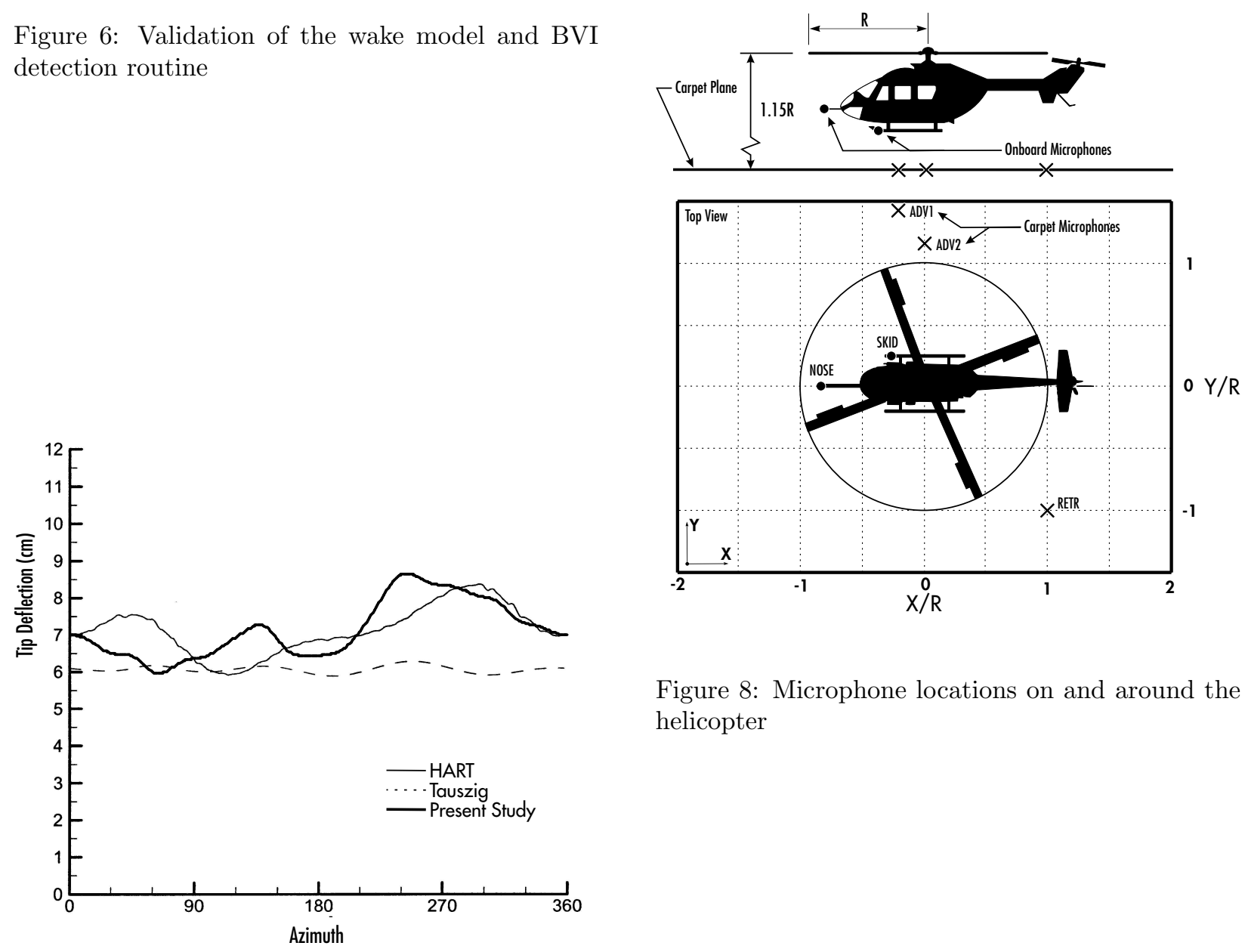

Figure 8: Microphone locations on and around the helicopter

Figure 7: Validation of the aeroelastic simulation by comparison of rotor tip deflections

American Institute of Aeronautics and Astronautics 


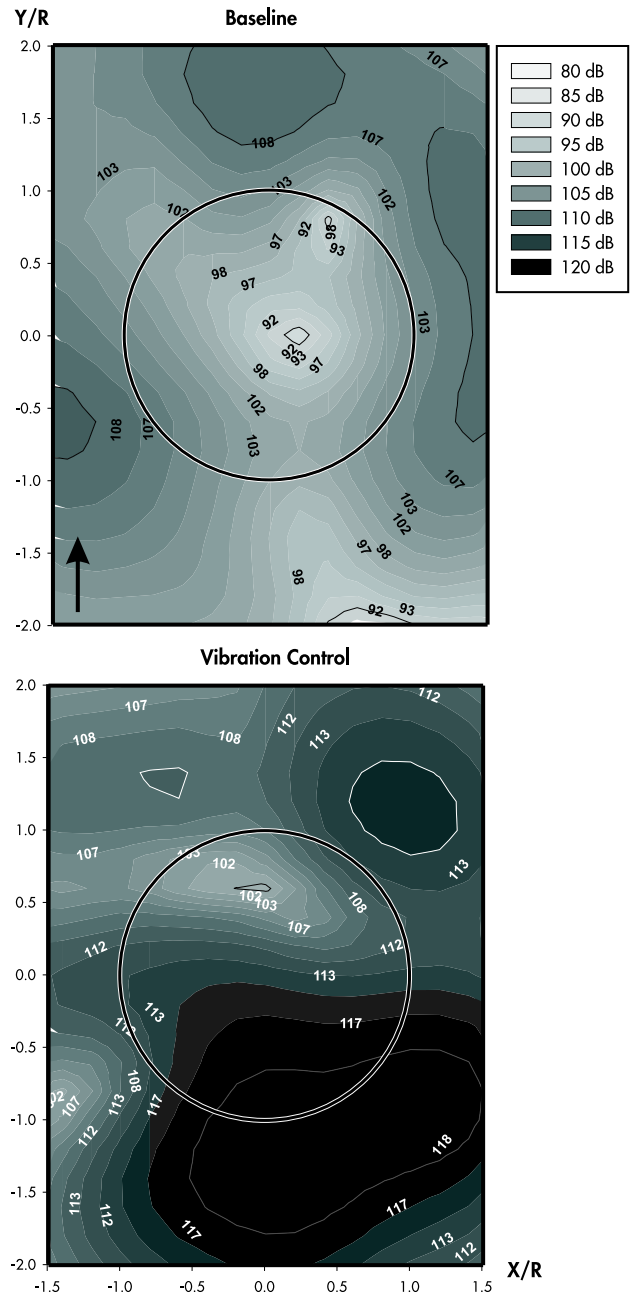

Figure 9: Carpet plots showing noise increase after vibration reduction

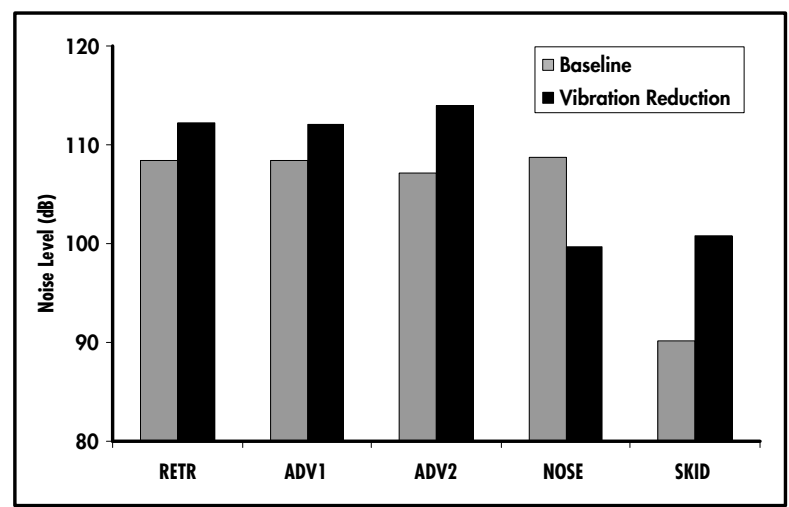

Figure 10: Effects of vibration reduction on noise levels for $\mu=0.15$

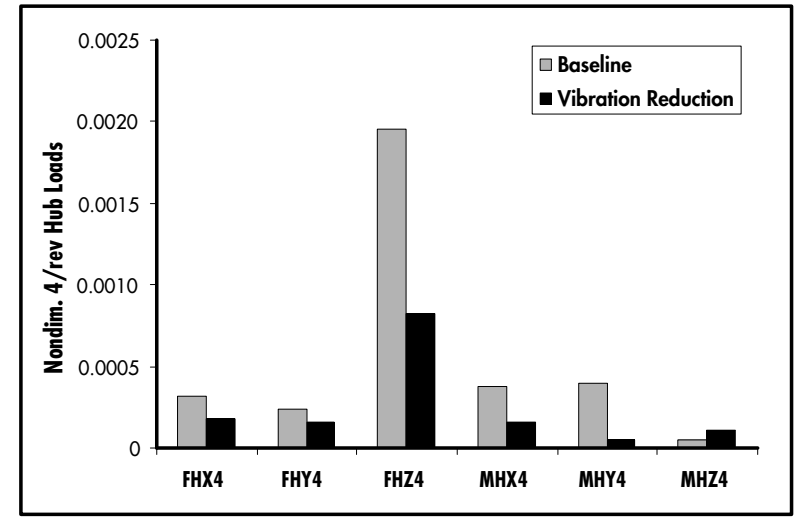

Figure 11: Reduction in vibration using an actively controlled flap for $\mu=0.15$

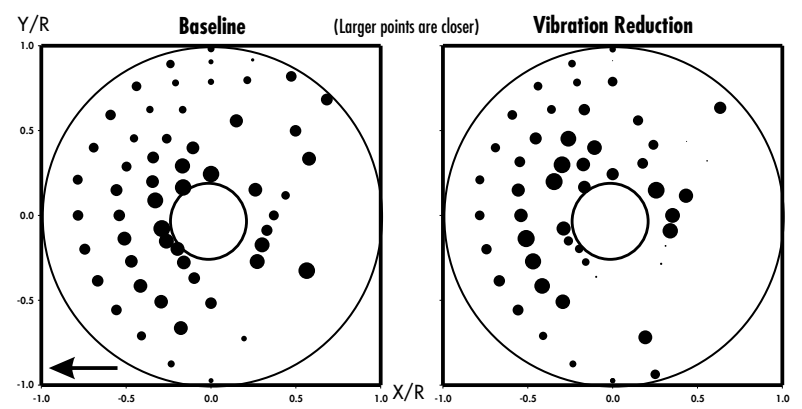

Figure 12: Effects of vibration reduction on bladevortex miss distance for $\mu=0.15$

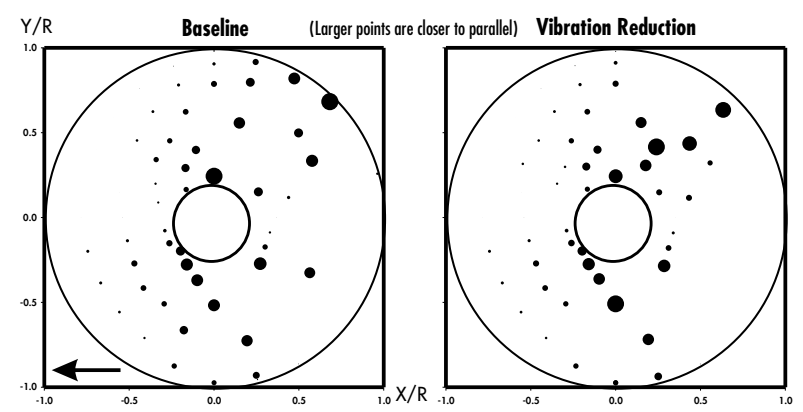

Figure 13: Effects of vibration reduction on bladevortex interaction angle for $\mu=0.15$ 


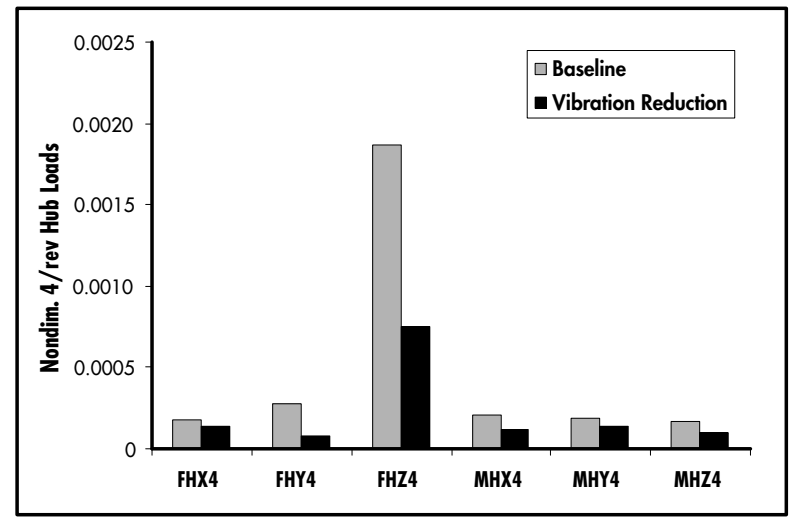

Figure 14: Reduction in vibration using an actively controlled flap for $\mu=0.30$

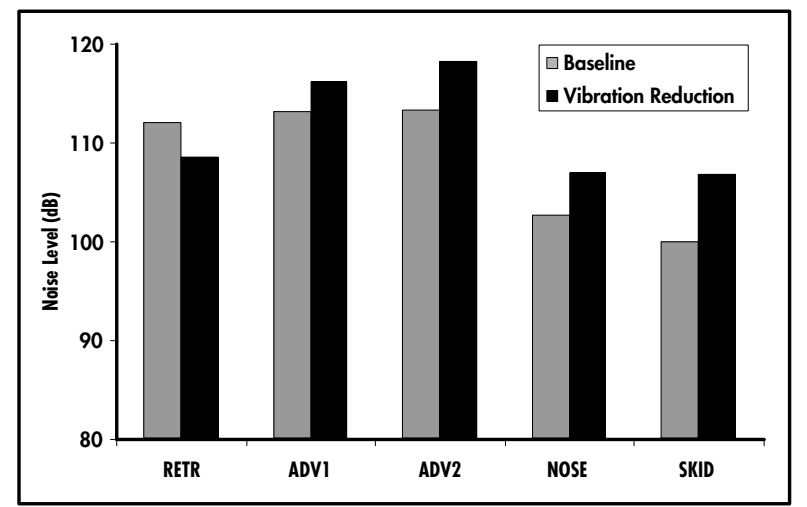

Figure 15: Effects of vibration reduction on noise levels for $\mu=0.30$

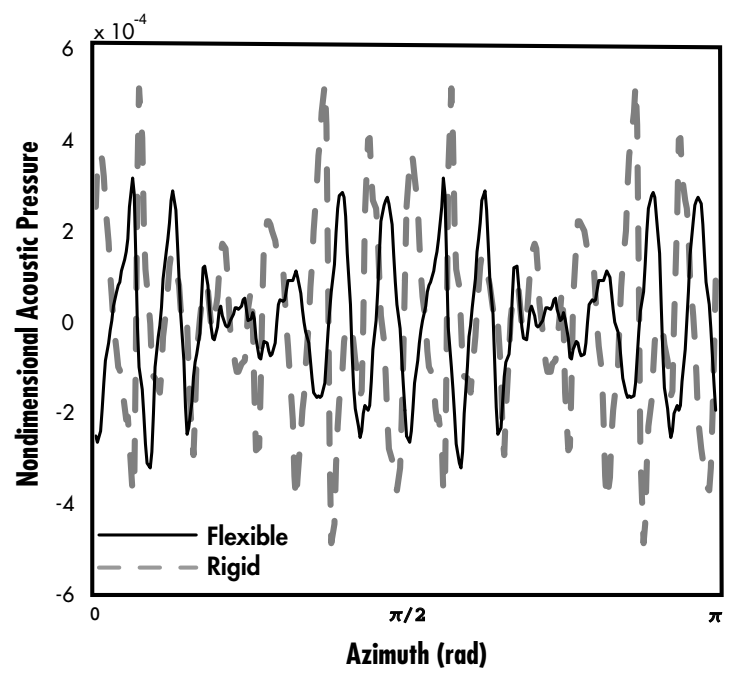

Figure 16: Effect of blade elasticity on noise signature $\mu=0.15$ 